\title{
Clinical Motion Tracking and Motion Analysis during Ophthalmic Surgery using Electromagnetic Tracking System
}

\author{
M. Ali Nasseri \\ Graduate School of \\ Information Science in Health \\ Technische Universität München \\ München, Germany
}

\author{
E. C. Dean, Suraj Nair \\ Martin Eder and Alois Knoll \\ Robotics and Embedded Systems \\ Technische Universität München \\ München, Germany
}

\author{
Mathias Maier \\ and Chris P. Lohmann \\ Augenklinik Rechts der Isar \\ Technische Universität München \\ München, Germany
}

\begin{abstract}
To design compact and precise robotic devices for medical applications one of the most important initial steps is choosing suitable actuators. In typical situations there is a tradeoff between working volume and precision. This trade-off and decision making is more serious in micro scale operations like ophthalmic surgeries. This paper analyzes the surgeons' motion during vitreo-retinal operation under clinical circumstances. The tracking system comprises an electromagnetic system to capture the surgical tool motion in 6 DOF and a coupled camera to keep the procedure under observation for the purpose of data validation. The contribution of this paper is divided to two folds: first is the precise definition of working volume and trajectories during ophthalmic surgery for robotic design optimization. This data will be used to define the parameters of actuators such as velocity, precision and travel range in design phase and will also be utilized for validation of the robot motion in further steps. The second contribution of this paper is the experimental setup which is extendable for observation of other micromanipulation applications such as ear surgery or cell injection.
\end{abstract}

\section{INTRODUCTION}

Eye surgeons around the world have to deal with everyday scenarios demanding high-precision manipulation of surgical devices. Surgeons' physiological tremor is about $108 \mu \mathrm{m}$ in amplitude reportedly [9] and can be dramatically increased by fatigue. Thus, most of the intraocular manipulations, which need micron scale tool motions, have to be performed by highly experienced and gifted surgeons. Finding a way to perform ophthalmology operations with fewer tremor and more precision can not only improve current clinical procedures, but will also open the door to new treatment options. Retinal Vein Occlusion (RVO) is an example of a serious sight-threatening retinal disease, which approximately 16 million people suffer from [7], and due to the human actuation and sensation limiting factors no clinical treatment has approved for this issue. This condition happens, when a clot is formed in one of the retinal veins or arteries which have a cross section diameter of $80 \mu \mathrm{m}$. One promising treatment is injecting clot-dissolving drugs like tissue Plasminogen Activator (tPA) directly into the blocked vessel [4]. Wei et al. proposed a method using stenting as a surgical treatment for Central Retinal Vein Occlusion (CRVO) [13]. Due to human physiological tremor, the procedure of locating the needle, and specifically holding the needle tip during the injection, is almost impossible to be performed by human surgeons.

The importance and complexity of RVO have attracted many robotics groups to develop a surgical assisted robot to perform this procedure. Untiring robots with excellent geometric accuracy, stability, and the ability of being designed to operate on many motion scales are suitable choices for performing retinal surgeries under demanding circumstances [11], [1], [12].

A proper surgical device for vitreo-retinal procedure should be as compact as possible, intuitive, and easy to setup in current operation theater domains as well as having the ability of fulfilling all the requirements in terms of tool motion and precision. To achieve these factors, a precise analysis of surgeon's motions during the operation is vital. Two methods

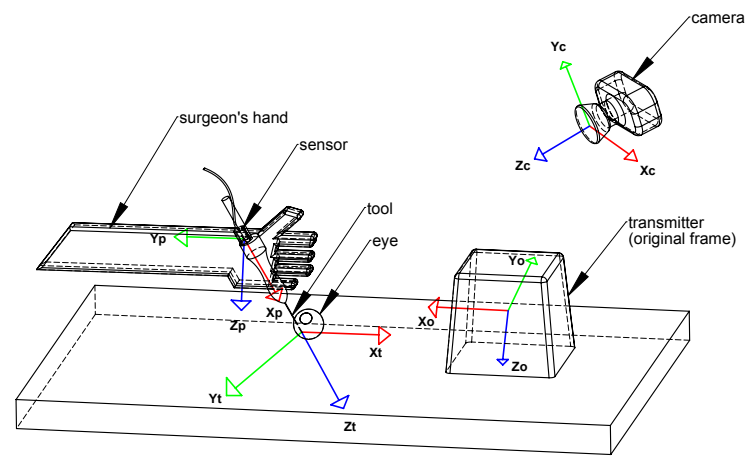

Fig. 1. Coordinate frames for the experimental setup, transmitter coordinate frame is used as the original coordinate frame for sensor and camera

for hand or tool motion tracking are Optical Tracking (OT) and Electromagnetic Tracking (EMT). In OT a rigid optical marker is attached to the tool or on the surgeon's glove and precise position and orientation of the marker are observed by multiple cameras. In EMT, an electromagnetic field generator is used near the surgical site and the relative position and orientation of a sensor, which is attached to the tool or glove, are observed with respect to the transmitter frame, see Figure 1. Although the precision of OT is better than EMT, a continuous visibility 


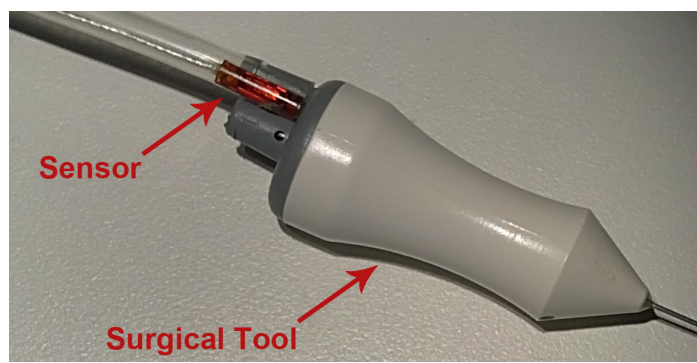

Fig. 2. Electromagnetic sensor mounted on vitrectomy tool

of the markers needs to be maintained during the procedure, i.e. occlusion must be avoided. Furthermore, the marker is not as small as EMT sensors, so the weight and length added to the tool are not negligible. Consequently, the motions during procedures which have limited movements with limited forces, such as in vitreo-retinal surgery, completely change in the presence of current OT markers. Thus, a promising alternative method for vitreo-retinal surgical tool tracking is EMT, where the visibility of the sensors is not a factor, and the sensor's weight and size are negligible.

In this work an electromagnetic tracking system is used to observe surgeon's motion during a real vitreo-retinal operation. The results of this work is a complete motion analysis of vitreo-retinal surgical tool in 6 DOF. Since the complete tracking data with relevant analysis during ophthalmology has not been collected before, this data can be used to optimize the design of a medical assisted device for vitreo-retinal surgery and simplify the trade-off between compactness and performance for engineers. The clinical data observed here will substitute the assumptions which currently are used in robotic designs and also serve as a toolkit for validation phase. This clinical data consists of working volume, tool position, tool orientation, angular and linear velocities, accelerations, trajectories and surgeon's emergency reaction. In design phase this data is useful to choose suitable actuators and in validation phase it is used as a reference for system behavior.

The organization of this paper is as follows: section 2 presents the methodology and experimental setup of this work with description of the tracking system, augmented reality and data acquisition. Section 3 summarizes results and discussion containing the analysis of the observed data in terms of positions, orientations and velocities. The safety and emergency motion is described in section 3 which will be followed by a conclusion in section 4 .

\section{Methodology And EXPERIMEnts}

\section{A. Tracking System}

To simulate the clinical environment as realistic as possible, the experimental setup was prepared in a surgeons' training room at the Klinikum Rechts der Isar ${ }^{1}$. Here the trainings are performed on a training table with a pig's eye, which is similar to human eye. The setup is exactly like in an operation on

\footnotetext{
${ }^{1}$ http://www.augenklinik.med.tum.de/
}

human eyes. The tracking system of this work comprises an electromagnetic tracking system, a "3D Guidance microBird" (Ascension Technology, USA) with mid-range transmitter and model $18 \mathrm{z}$ sensors. This system captures motion with $0.8 \mathrm{~mm}$ and 0.5 degrees precision which is acceptable for the objective of this study. The optimal tracking volume for this combination is:

- $X=20$ to $51 \mathrm{~cm}, Y= \pm 23 \mathrm{~cm}$ and $Z= \pm 15 \mathrm{~cm}$ from the transmitter center (see Figure 1)

The transmitter was placed on the table close to the head in the optimal tracking area (see Figure 3). As shown in Figure 2 the sensor placed on the instrument did not interfere with the handling, and the movement by the surgeon was not affected in any way. In order to maintain optimum tracking accuracy a non-metallic table was used. The DC EM tracking system is theoretically susceptible to metals with high magnetic permeability [5], but in our application such effects were minimized. In order to have a visual supervision over the experiments a 1.3 megapixel camera was added to the tracking system in an appropriate location to cover the surgical area (see Figure 3). The idea of combining the information observed with the electromagnetic system and the images taken by camera was to complete the tracking procedure, which later will be used as a feedback on data validation and a suitable way of motion visualization. First the complete hardware setup, including placement of the camera and the transmitter in suitable locations was prepared. In the next step an expert surgeon was asked to prepare the pig's eye for operation, followed by the procedures containing the adjustment of the microscope, inserting the trocars (23 Gauge trocar system was used) and connecting the infusion line. Then he was asked to perform an operation while the tracking software was running. The tracking software developed for this study gathers the information of the position $(X, Y, Z$ in millimeter) and orientation (Azimuth $(A)$, Elevation $(E)$, and $\operatorname{Roll}(R)$ in degrees), adds timestamps information, and saves all information continuously in a file during the procedure.

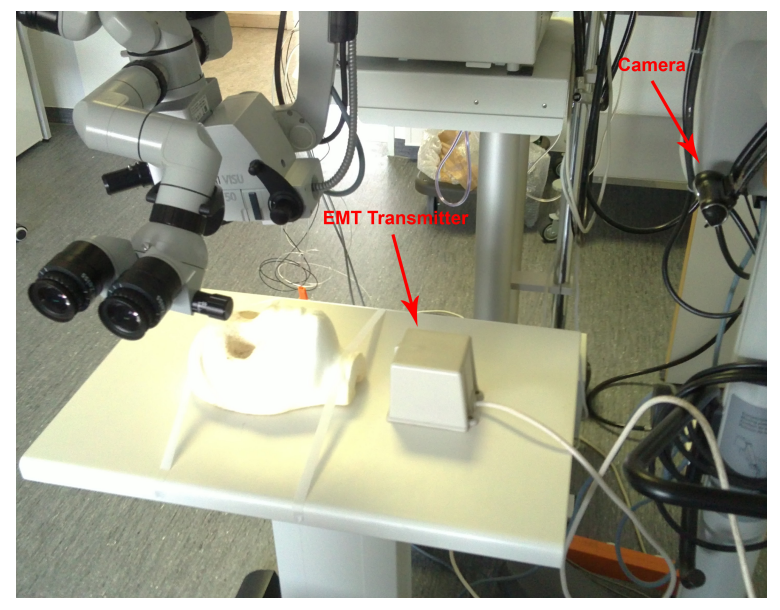

Fig. 3. Experimental setup with operating table, microscope, electromagnetic transmitter, camera in a proper location with respect to the head 


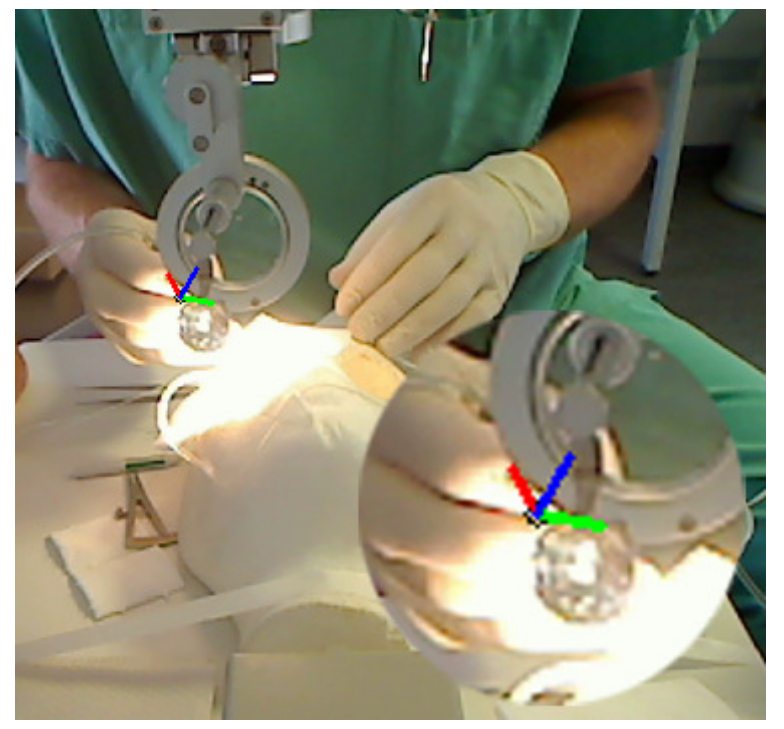

Fig. 4. In each captured frame the position of sensor is marked with a black dot and the orientation of the sensor is represented by a coordinate frame

At each time stamp the data contains:

- An ID for each data point. The image captured by the camera for each data has the same ID for further references.

- $X, Y$ and $Z$ in millimeters, representing the relative position of sensor with respect to the transmitter frame shown in Figure 1.

- $A, E$ and $R$ in degrees, representing the relative orientation of sensor with respect to the transmitter frame shown in Figure 1 in degrees.

- A time stamp in seconds, representing when the data was captured.

In this study the exact position of the EMT sensor was desired, otherwise there was a possibility to analyze any point along the medical tool (e.g. tool tip) by simple homogeneous transformation.

\section{B. Augmented Reality}

In order to complete the interaction loop during surgery and to have a visual feedback during the procedure, a camera was added to our tracking system. The camera was calibrated to project a 3D point (sensor position), which is known with respect to EMT transmitter shown in Figure 3. By setting the transmitter frame as the original frame for EMT system and the camera, the position and the orientation of the sensor could be projected onto the image. the projection was performing as follows, The projection matrix was defined as:

$$
P=\overbrace{\left[\begin{array}{cccc}
f_{x} & 0 & c_{x} & 0 \\
0 & f_{y} & c_{y} & 0 \\
0 & 0 & 1 & 0
\end{array}\right]}^{\text {intrinsic }} \overbrace{\left[\begin{array}{cccc}
R_{1,1} & R_{1,2} & R_{1,3} & t_{x} \\
R_{2,1} & R_{2,2} & R_{2,3} & t_{y} \\
R_{3,1} & R_{3,2} & R_{3,3} & t_{z} \\
0 & 0 & 0 & 1
\end{array}\right]}^{\text {extrinsic }}
$$

The $P$ matrix is a 3 by 4 matrix defined by multiplication of intrinsic matrix by extrinsic matrix. Where the intrinsic matrix represents the intrinsic camera parameters, which depend only on camera characteristics such as focal length $f$, pixel size and principal points. The Extrinsic matrix included the current position and orientation of the camera, and defines the rotation matrix and translation vector of camera with respect to a known frame that in this case is EMT transmitter center frame. Both intrinsic and extrinsic matrices were extracted by a camera calibration procedure using a calibration pattern with known 3D geometry [14]. Next, for projecting the 3D position of the sensor into the current image the following computation was used:

$$
\left[\begin{array}{c}
x_{c} \\
y_{c} \\
1
\end{array}\right]=P\left[\begin{array}{c}
x_{p} \\
y_{p} \\
z_{p} \\
1
\end{array}\right]
$$

where $x_{c}$ and $y_{c}$ are pixel coordinates and define the position of the sensor on each image. For projecting the orientation of the sensor, the rotation matrix was used to visualize the coordinate frame of the sensor on each frame with respect to its relevant $A, E$ and $R$ angles. In order to track all the orientations without having singularities the quaternion based rotation representation was used. For projecting coordinate frames of the sensor on the image the following technique was implemented:

$$
\overbrace{\left[\begin{array}{ccc}
x_{x} & x_{y} & x_{z} \\
y_{x} & y_{y} & y_{z} \\
z_{x} & z_{y} & z_{z}
\end{array}\right]}^{X_{\text {axis }}\left|Y_{\text {axis }}\right| Z_{\text {axis }}}=R\left[\begin{array}{ccc}
d & 0 & 0 \\
0 & d & 0 \\
0 & 0 & d
\end{array}\right]
$$

where $d$ is the length of the axis (in pixels), which will be presented on the image. By projection of $X_{\text {axis }}, Y_{\text {axis }}$ and $Z_{\text {axis }}$ and connecting this point to the center point, positions and orientations of the sensor connected to the surgical tool were visualized. In Figure 4 a frame captured during the procedure is presented.

\section{Data Acquisition}

With the aim of getting more realistic data, the surgeon was asked to perform the operation three times and the raw data for all motions were captured. Then the raw data was analyzed in terms of total working volume, positions, velocities, accelerations and trajectories. The total duration of operation was 5 minutes in three phases: Approaching the tool-tip to the eye, insertion of the tool tip into the eye using trocar canals, and tool manipulation inside the eye. In total around 12000 data entries were captured to cover all possible motions during tool manipulation inside the eye. Here, 70 seconds during vitrectomy performance were analyzed. Vitrectomy procedure was used because the procedure of removing vitreous from the eye (vitrectomy) has the most significant motions among all the intraocular operations.

\section{RESUlts AND Discussions}

Tool positions can be seen in Figure 5 and Figure 6 during the vitrectomy of the pig's eye. To simplify the visualizations, the insertion point of the tool-tip was chosen as the 


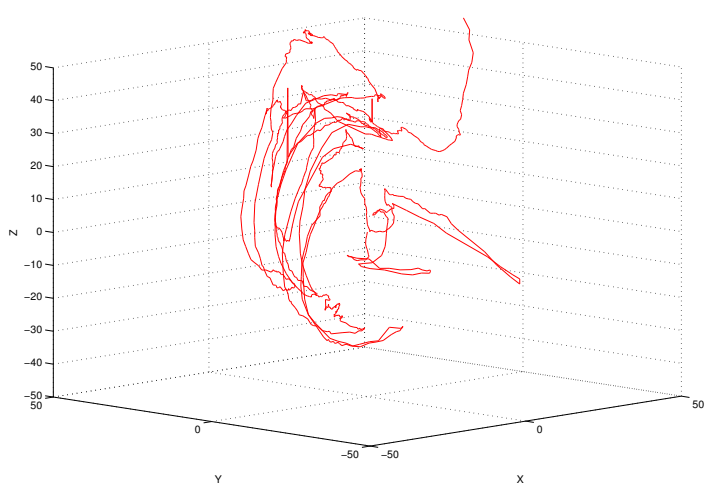

Fig. 5. Tool trajectory in 3D space

refrence point and the position and the orientation values were visualized with respect to insertion point. In Table I the information observed from raw data, including minimum and maximum position of tool and motion ranges for each axis is summarized. For this procedure the working volume of the surgeon can be defined as $47 \times 63 \times 97 \mathrm{~mm}$ (see Figure 5). An interesting fact, which can be observed from these graphs, is that the motion in $X$ and $Y$ directions is minimal, while the $Z$ is less than $-27 \mathrm{~mm}$ (see Figure 6). In other words the tool tip movement will decrease, when it's getting close to the retina.
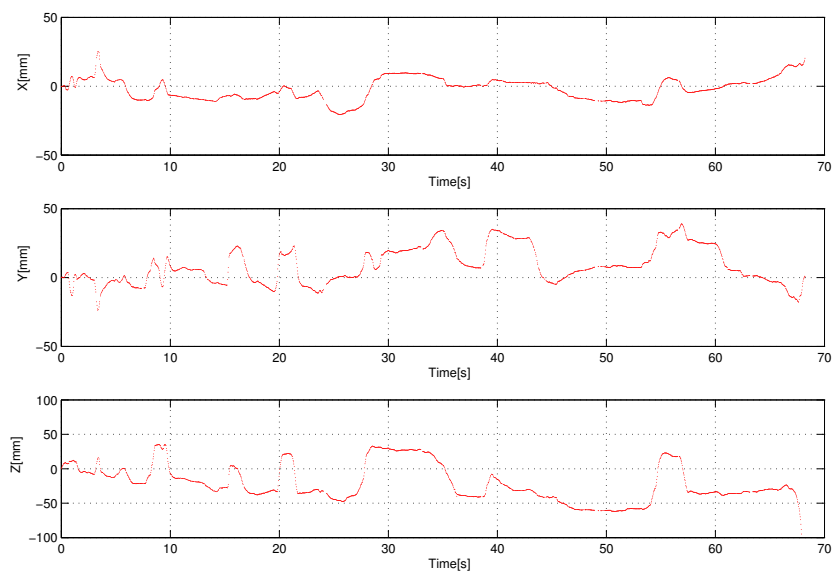

Fig. 6. Tool position during a major procedure in $x$ (1st plot), $y$ (2nd plot) and $z$ (last plot) axes

Figure 7 represents the tool motion in terms of orientations in this procedure. The maximum, minimum, and the range of angular motion in different directions can be seen in Table I.

TABLE I

POSITIONS AND ORIENTATIONS CHANGE DURING VITRECTOMY ON PIG'S EYE

\begin{tabular}{ccccccc}
- & $\mathrm{X}$ & $\mathrm{Y}$ & $\mathrm{Z}$ & $\mathrm{A}$ & $\mathrm{E}$ & $\mathrm{R}$ \\
\hline \hline Min & $-21 \mathrm{~mm}$ & $-24 \mathrm{~mm}$ & $-61 \mathrm{~mm}$ & $-36^{\circ}$ & $-50^{\circ}$ & $-114^{\circ}$ \\
Max & $+26 \mathrm{~mm}$ & $+39 \mathrm{~mm}$ & $+36 \mathrm{~mm}$ & $+16^{\circ}$ & $+22^{\circ}$ & $+35^{\circ}$ \\
Range & $47 \mathrm{~mm}$ & $63 \mathrm{~mm}$ & $97 \mathrm{~mm}$ & $52^{\circ}$ & $72^{\circ}$ & $149^{\circ}$
\end{tabular}

According to the observation from angular and the linear motions of ophthalmic surgery, the angular motion about $X$ and $Y$ and linear motion of these axes are changing together which is the consequence of Remote Center of Motion (RCM) constraint. This constraint forces the surgeon to pivot the tool about the insertion point which is the trocars inserted on the surface of sclera. But the rotation around the tool axis $(Z)$ is independent of the linear motion along $Z$.
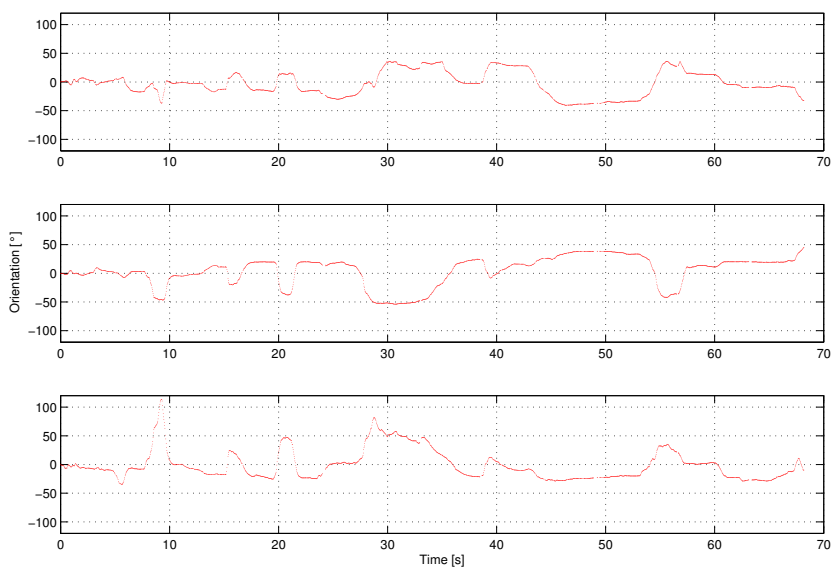

Fig. 7. Tool Orientation during a major procedure $A(1$ st Plot), $E$ (2nd Plot) and $R$ (Last Plot)

Figure 8 shows the linear velocities of the tool. The maximum velocity along $X$ and $Y$ direction is $20 \frac{\mathrm{mm}}{\mathrm{s}}$ and along $Z$ direction is $40 \frac{\mathrm{mm}}{\mathrm{s}}$, but the average velocities are less than $5 \frac{\mathrm{mm}}{\mathrm{s}}$. Another observation from velocity diagram is the hand tremor of the surgeon in different directions which can be observed more specifically than within position diagram. This effect is consequence of moving to the higher order domain. But the EMT method is not suitable to measure surgeon's tremor precisely which according to the state of the art reported less than $200 \mu \mathrm{m}$ [1].

Figure 9 is the diagram of angular velocities in $A, E$ and $R$ directions. The maximum angular velocity around $X$ is $20 \frac{\text { degrees }}{\mathrm{s}}$, around $Y$ is $25 \frac{\text { degrees }}{\mathrm{s}}$ and around $Z$ is $40 \frac{\text { degrees }}{\mathrm{s}}$ the
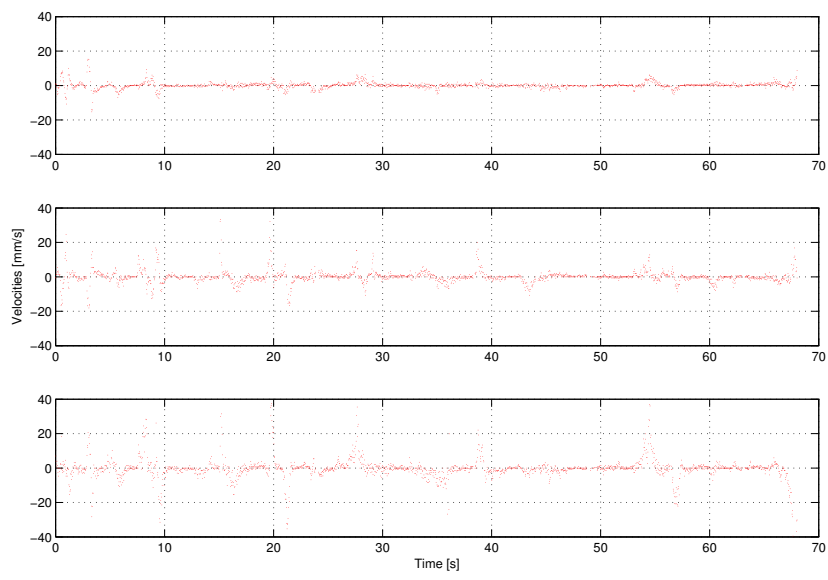

Fig. 8. Linear velocities in $X, Y$ and $Z$ directions 

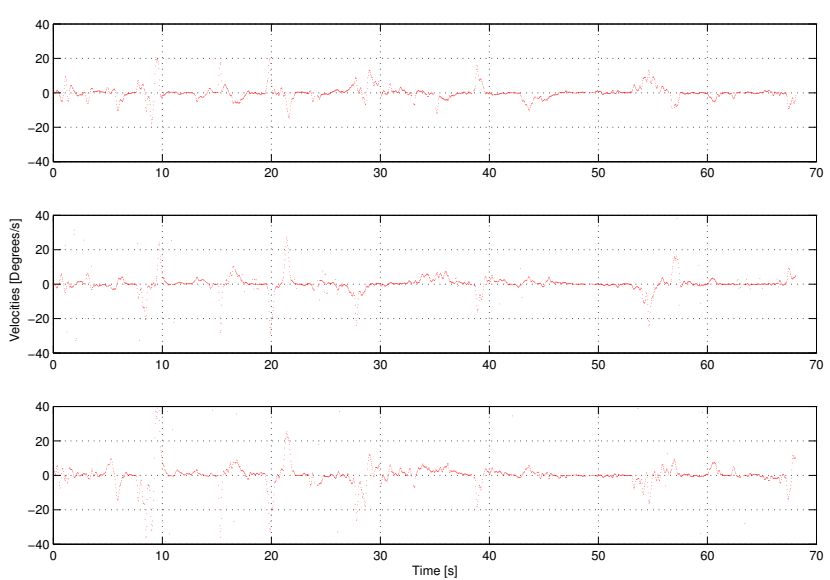

Fig. 9. Angular velocities about $X$ (Azimuth), $Y$ (Elevation) and $Z($ Roll $)$

reason of more angular velocity around $Z$ is the less risk of tool rotation around its axis. Like the diagram of linear velocity, in figure 9 surgeon's tremor during angular motions is visible.

\section{A. Safety and Emergency}

Figure 10 represents the velocity of the tool in $Z$ direction when the surgeon wants to approach the retina. In the left plot the $Z$ motion of the tool can be seen, right plot shows the $Z$ velocity for the same motion. The comparison of these two plots clearly shows that velocity in $Z$ direction is inversely related to the tool-tip distance from the retina. This is due to safety reasons, the surgeon tries to avoid contact with retinal surface. This condition is important when no intraocular force feedback is available and the surgeon should rely on visual feedback from microscope.

The emergency procedure in retinal surgery is to remove the tool along the approach axis without any other angular or linear motion. Therefore it is necessary to design a surgical device or robot for assisting surgeons to perform retinal surgery, having independent motion along approach axis in the end-effector.
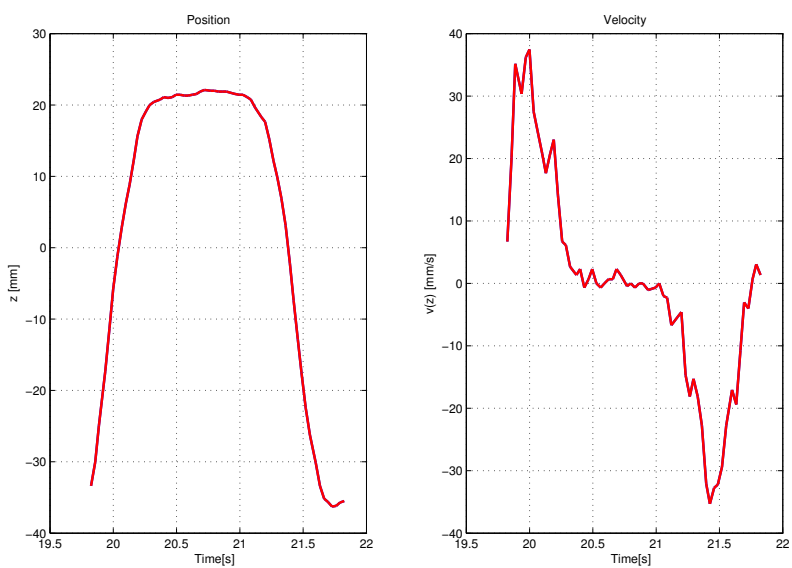

Fig. 10. Velocity in $Z$ direction while surgeon approaches the retina; left plot is position and right plot is velocity

\section{CONCLUSION}

In this study the surgical motion during vitreo-retinal surgery was clinically captured and analyzed. For this purpose, an experimental setup with a combination of an electromagnetic tracking system and a camera was developed. During the experiments the precise motion of an expert surgeon in $6 \mathrm{DOF}$ was observed and determined. The outcome of this study is as follows: The Experimental Data contain trajectory and working volume of the tool, position analysis, orientation analysis, linear and angular velocities of tool during the procedures and emergency reaction by the surgeon. This information which has not collected before will be used to design robotic devices with the aim of performing vitreo-retinal surgery also it is a clinical reference for validation purposes.

\section{REFERENCES}

[1] Ang, W.T., Pradeep, P.K., Riviere, C.N.: Active tremor compensation in microsurgery. In: Eye . pp. 2738-2741 (2004)

[2] Hubschman JP, Son J, Allen B, Schwartz SD, Bourges JL.: Evaluation of the motion of surgical instruments during intraocular surgery. In: Proceedings of the 26th Annual International Conference of the IEEE EMBS'. pp. 947-953 (2011)

[3] Aschke, M., Wirtz, C., Raczkowsky, J., Wrn, H., Kunze, S.: Stereoscopic augmented reality for operating microscopes. In: Proceedings of Int'l Congress on Computer Assisted Radiology and Surgery. pp. 666-673 (2003)

[4] Bynoe, L., Hutchins, R., Lazarus, H., Friedberg, M.: Retinal endovascular surgery for central retinal vein occlusion: initial experience of four surgeons. Retina 25, 625-632 (2005)

[5] Hummel, Bax, J.B., Figl, M.R., Kang, M.L., Maurer, Y., Birkfellner, J.C., Bergmann, W.W., Shahidi, R.: Design and application of an assessment protocol for electromagnetic tracking systems. Medical Physics 32, 2371-2379 (2005)

[6] Reiley, C.E., Chen, C.C.G., Hager, G.D.: Skill assessment for robotic surgery using statistical models: Language of surgery. American Urogynecologic Society (AUGS) (2010),

[7] Rogers, S., Rachel, L.M., Cheung, N., Lim, L., Wang, J., Mitchell, P., Kowalski, O.W., Nguyen, H., Wong, T.Y.: The prevalence of retinal vein occlusion: Pooled data from population studies from the United states, Europe, Asia, and Australia. Ophthalmology 117, 313-319 (2010)

[8] Saleh, G., Voyatzis, G., Hanse, J., Darzi, A.: Evaluating surgical dexterity during corneal suturing. Arch Ophthalmol 124, 1263-1266 (2006)

[9] Sinch, S., Riviere, C.: Physiological tremor amplitude during retinal microsurgery. In: IEEE Northeast Bioengineering Conference. pp. 171172 (2002)

[10] Sznitman, R., Basu, A., Richa, R., Handa, J., Gehlbach, P., Taylor, R.H., Jedynak, B., Hager, G.D.: Unified detection and tracking in retinal microsurgery. In: Fichtinger, G., Martel, A., Peters, T. (eds.) Medical Image Computing and Computer-Assisted Intervention - MICCAI 2011. Lecture Notes in Computer Science, vol. 6891, pp. 1-8. Springer (2011)

[11] Ueta, T., Yamaguchi, Y., Shirakawa, Y., Nakano, T., Ideta, R., Noda, Y., Morita, A., Mochizuki, R., Sugita, N., Mitsuishi, M., Tamaki, Y.: Robot-assisted vitreoretinal surgery: Development of a prototype and feasibility studies in an animal model. Ophthalmology 116, 1538-1543 (2009)

[12] Uneri, A., Marcin, A., Balicki, J., Handa, P., Gehlbach, Taylor, R., Iordachita, I.: Precision experiments on a comanipulated robotic system for use in retinal surgery. In: Proceedings of the 2011 SCATh Joint Workshop on New Technologies for Computer/Robot Assisted Surgery.2. pp. 1-7 (2011)

[13] Wei, W., Popplewell, Fine, C., Chang, H.F., Simaan, N.: Enabling technology for microvascular stenting in ophthalmic surgery. ASME Journal of Medical Devices 4, 014503(1-6) (2010)

[14] Zhang, Z.: Flexible camera calibration by viewing a plane from unknown orientations. In: The Proceedings of the Seventh IEEE International Conference on Computer Vision. pp. 408-413 (1999) 